\title{
Neighbor-Finding Based on Space-Filling Curves ${ }^{1}$
}

\author{
Hue-Ling Chen, and Ye-In Chang \\ Dept. of Computer Science and Engineering \\ National Sun Yat-Sen University \\ Kaohsiung, Taiwan \\ Republic of China \\ \{E-mail: changyi@cse.nsysu.edu.tw\} \\ \{Tel: 886-7-5252000 (ext. 4334)\}
}

\{Fax: 886-7-5254301\}

\begin{abstract}
Nearest neighbor-finding is one of the most important spatial operations in the field of spatial data structures concerned with proximity. Because the goal of the space-filling curves is to preserve the spatial proximity, the nearest neighbor queries can be handled by these space-filling curves. When data is ordered by the Peano curve, we can directly compute the sequence numbers of the neighboring blocks next to the query block in 8 directions in the $2 D$-space based on its bit shuffling property. But when data is ordered by the RBG curve or the Hilbert curve, neighbor-finding is complex. However, we observe that there is some relationship between the RBG curve and the Peano curve, as with the Hilbert curve. Therefore, in this paper, we first show the strategy based on the Peano curve for the nearest neighbor query. Next, we present the rules for transformation between the Peano curve and the other two curves, including the RBG curve and the Hilbert curve, such that we can also efficiently find the nearest neighbor by the strategies based on these two curves. From our simulation, we show that the strategy based on the Hilbert curve requires the least total time (the CPU-time and the I/O time) to process the nearest neighbor query among our three strategies, since it can provide the good clustering property.
\end{abstract}

(Key Words: Hilbert curve, neighbor-finding, Peano curve, RBG curve, space-filling curves, spatial proximity.)

\footnotetext{
${ }^{1}$ Recommended by Hue-Ling Chen, and Ye-In Chang
} 


\section{Introduction}

With the proliferation of wireless communications and the rapid advances in GIS systems, a very common spatial query is the "Nearest Neighbor Query" which seeks for the objects residing more closely to a given object. For example, when driving a car in a highway, the driver may ask where the nearest gas station is. In other words, nearest neighbor-finding is one of the most important spatial operations in the field of spatial data structures regarding proximity.

Basically, spatial data consists of objects in space made up of points, lines, regions, rectangles, and data of higher dimensions. Access methods are required to support efficient manipulation of the multi-dimensional spatial objects in the secondary storage. Several schemes $[2,3,4,8,12,13,14]$ for the linear mapping of a multi-dimensional space have been proposed for various applications, such as access methods for traditional databases, geographic information systems (GIS) and spatio-temporal databases. However, in spatial applications, the structure of databases become larger and more complex and means for extracting valuable information become more sophisticated [8]. The design of multidimensional access methods is difficult compared to one-dimensional cases because there is no total ordering that preserves spatial locality [12]. Once a total ordering is found for a given spatial database, one can use any one-dimensional access method which may yield good performance for multi-dimensional queries. Thus, what is needed is a mapping from a higher dimensional space to a one-dimensional space. In this mapping, the elements which are close in space are mapped into nearby points on the line to preserve the spatial locality. A space-filling curve passes through every point in the space once. It is one kind of mapping to give an one to one correspondence between the coordinates and the sequence numbers of the points on the curve [8].

Because the goal of the space-filling curves is to preserve spatial proximity, they can handle nearest neighbor queries. Some examples of space-filling curves are the Peano curve, the RBG curve and the Hilbert curve. The Peano curve $[13,14]$ (or the $Z$-order curve) has the bit shuffling property, which means interleaving the bits from two coordinates of the point in base 2 in the $2 D$-space. An improvement of the Peano curve is the Reflected Binary Gray-code $(R B G)$ curve $[2,3]$ which uses Gray coding on the interleaved bits to 
reduce random accesses on the disk for range queries. Based on the Peano curve, the Hilbert curve was proposed with superior data clustering properties, which means that the locality between objects in the multi-dimensional space is preserved in the linear space $[4,6,7,8,12]$.

When the data is linearly ordered by the Peano curve, we can directly compute the sequence numbers of the neighboring blocks next to the query block in 8 directions based on its bit shuffling property, even the order is greater than 1. (Note that the order of a curve deals with how many sequence numbers be necessary to uniquely define that curve.) But with the data ordered by the RBG curve or the Hilbert curve, neighbor-finding would be complex. However, we also observe that there is some relationship between the RBG curve and the Peano curve, as with the Hilbert curve. Therefore, in this paper, we first show how these space-filling curves are derived and discuss the relationships between them. Next, we describe the effects on nearest neighbor queries by using different curves. Then, we show the way to find the nearest neighbor by our strategy based on the Peano curve, and present the rules for transformation between the Peano curve and others. Finally, we compare the performance of our strategy for the nearest neighbor-finding based on different space-filling curves.

The rest of paper is organized as follows. In Section 2, we briefly describe three spacefilling curves, the Peano curve, the RBG curve, the Hilbert curve, and their spatial properties. In Section 3, we present our strategies for the nearest neighbor-finding, based on the Peano curve and the transformation rules between the Peano curve and the others. In Section 4, we describe the simulation experiment performed to compare different strategies, and results obtained therefrom. Finally, we provide conclusions for this paper.

\section{Space-Filling Curves}

The design of multi-dimensional access methods is difficult compared to one-dimensional cases because there is no total ordering that preserves spatial locality [12]. One way is to look for a mapping which preserves spatial proximity at least to some extent. In this section, we discuss the properties of the Peano curve, the RBG curve, and the Hilbert curve. 


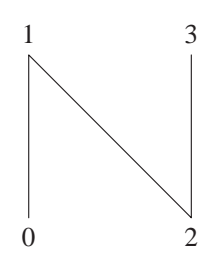

(a)

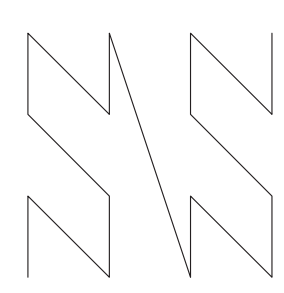

(b)

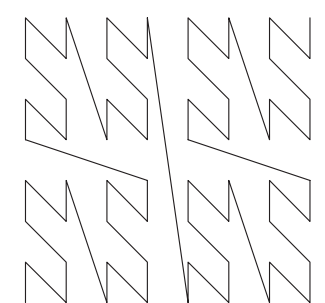

(c)

Figure 1: Peano curves of order: (a) 1; (b) 2; (c) 3.

\subsection{Properties}

A space-filling curve orders points linearly to preserve the distance between two points in the $2 D$-space. This means that points which are close in space and represent similar data should be stored together in the linear sequence. Some examples of space-filling curves are the Peano curve, the RBG curve and the Hilbert curve $[2,3,4,6,7,12,13,14]$. In general, a space-filling curve starts with a basic path on a $k$-dimensional square grid of side 2 . The path visits every point in the grid exactly once without crossing itself. It has two free ends which may be joined with other paths. The basic curve is said to be of order 1 . To derive a curve of order $i$, each vertex of the basic curve is replaced by the curve of order $i-1$, which may be appropriately rotated and/or reflected to fit the new curve [4].

For the Peano curve, the $1 D$-number (one-dimensional sequence number) of a point is obtained by bit shuffling on the $X$ and $Y$ coordinates of the point in the $2 D$-space (twodimensional space). The basic Peano curve of order 1 for a $2 \times 2$ grid, denoted as $P_{1}$, is shown in Figure 1-(a). To derive higher orders of the Peano curve, we replace each vertex of the basic curve with the previous order curve. Figures 1-(b) and (c) show the Peano curves of order 2 and 3 , respectively $[4,6]$.

For the RBG curve, numbers are coded into binary gray-codes such that successive numbers differ in exactly one bit position. Faloutsos [2, 3, 4] observed that in the RBG curve, the difference in only one bit position has a relationship with locality. He proposed a formula to transform the Peano curve into the RBG curve such that the property of locality can be preserved. The basic RBG curve of order 1 for a $2 \times 2$ grid, denoted as $R_{1}$ is shown in Figure 2-(a). Higher orders of this curve are derived by reflecting the previous order 


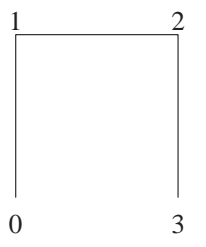

(a)

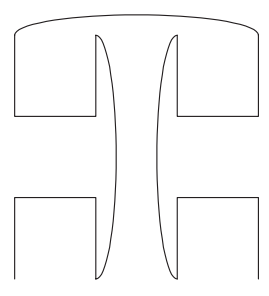

(b)

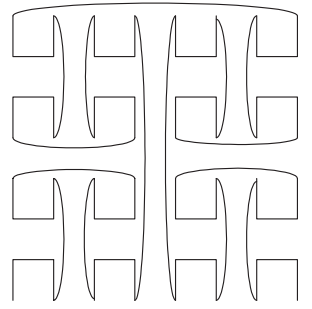

(c)

Figure 2: RBG curves of order: (a) 1; (b) 2; (c) 3.

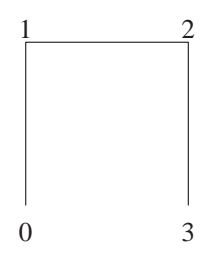

(a)

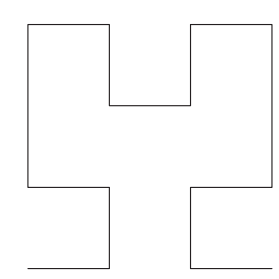

(b)

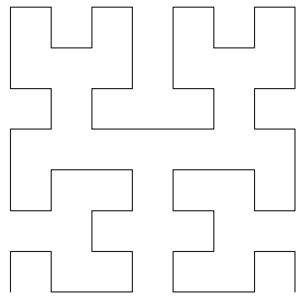

(c)

Figure 3: Hilbert curves of order: (a) 1; (b) 2; (c) 3.

curve over $x$-axis and then over the $y$-axis. Figures 2-(b) and (c) show the RBG curve of orders 2 and 3 , respectively $[2,3,4,6]$.

The Hilbert curve is a space-filling curve used to preserve spatial locality as much as possible. Thus, the Hilbert curve has the important property that consecutively ordered points are adjacent in space [8], as shown in Figure 3-(a). As in the case of the previous two curves, it replicates in four quadrants. While replicating, the lower left quadrant is rotated clockwise $90^{\circ}$, the lower right quadrant is rotated anti-clockwise $90^{\circ}$, and the sense (the direction of traversal) of both lower quadrants are reversed. The two upper quadrants do not rotate and change the sense. Thus we obtain Figure 3-(b). Because all rotation and sense computations are relative to previously obtained ones in a particular quadrant, a repetition of this step gives rise to Figure $3-(\mathrm{c})[2,3,4,6,7]$.

Space-filling curves are based on the assumption that any attribute value can be represented with some fixed number of bits. In the $2 D$-space, there are two attributes: the horizontal and vertical directions which are called the $X$ and $Y$ axes, respectively. The whole space is split into equal-sized sub-regions, and the direction of splitting alternates 
between $X$ and $Y$ axes. A vertical split through the middle of the space amounts to discrimination in the value of $x_{0}$. If the resulting sub-regions are split horizontally, this corresponds to discrimination on the value of $y_{0}$. At this point, there are four equal-sized sub-regions, corresponding to the possible values of $x_{0}$ and $y_{0}$.

In general, each split on $X$ or $Y$ axis is characterized by one bit. After splitting $k$ times in each direction, a grid can be specified by $2 k$ bits of two coordinates, $\left(x_{k-1} x_{k-2} \ldots x_{1} x_{0}\right.$, $\left.y_{k-1} y_{k-2} \ldots y_{1} y_{0}\right)_{2}$. The bit string obtained by bit shuffling on these $2 k$ bits uniquely identifies one sub-region. The $z$-value is represented as the decimal value of the bit string. This means that there is a concise description of the shape, size and position of the sub-region. All points inside the sub-region have the same coordinates as the sub-region [14]. Consider the point or the region with $X=(1)_{10}=(\underline{0} \underline{1})_{2}=\left(x_{1} x_{0}\right)_{2}$ and $Y=(3)_{10}=(11)_{2}=\left(y_{1} y_{0}\right)_{2}$ in Figure 4-(a), where ( $)_{2}$ represents the binary form of the number, and ( $)_{10}$ represents the decimal form of the number. The point or the region has the bit string $(\underline{0} 1 \underline{11})_{2}=$ $\left(x_{1} y_{1} x_{0} y_{0}\right)_{2}$, obtained by bit shuffling, which represents the splitting process on $X$ and $Y$ axes. In the decimal representation, the $z$-value of the bit string $(\underline{0} 1 \underline{11})_{2}$ is equal to $(7)_{10}$. Orenstein $[13,14]$ used the $z$-values and $z$-ordering to refer to the ordering of the Peano curve (Figure 4-(a)), which is a total ordering of elements that combines with the highly constrained splitting policy and preserves proximity. Similarly, $r$-ordering and $r$-values are used for the RBG curve, and $h$-ordering and $h$-values are used for the Hilbert curve, as shown in Figures 4-(b) and (c), respectively.

\section{$3 \quad$ Nearest Neighbor-Finding}

Nearest neighbor-finding is one of the most important spatial operations in the field of spatial data structures which is concerned with proximity $[1,5]$. Because the goal of the space-filling curves is to preserve spatial proximity, nearest neighbor queries can be handled by the space-filling curves. An example of the nearest neighbor query shown in Figure 5 is to find the closest hydrant to the point of a fire by using three different curves. The entire grid is a map of a neighborhood, which is stored in a database. There exists $F$, which represents the point of the fire, and $H$, which represents a hydrant in the area. The lines represent street blocks and the numbers represent the orders that are stored in 


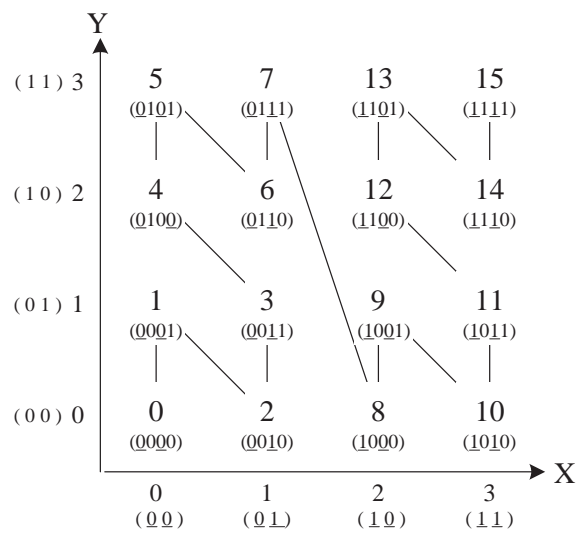

(a)

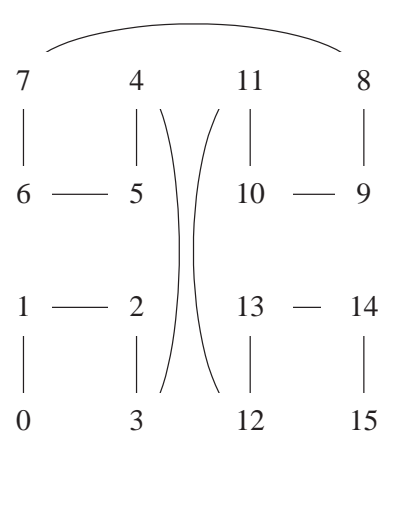

(b)

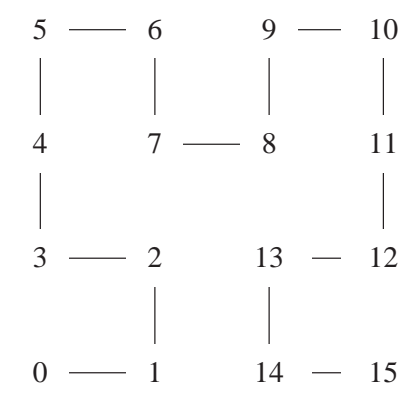

(c)

Figure 4: Space-filling curves of order 2: (a) z-ordering: Peano curve; (b) $r$-ordering: RBG curve; (c) h-ordering: Hilbert curve.

the database. In order to find the nearest data point $H$ to the query point $F$, we briefly describe the conventional approach as follows.

In the conventional approach [4], we first compute the sequence number of $F$, and find $H$ which has the closest sequence number (either in the preceding or succeeding order) to that of $F$ along the curve path. Then, we calculate the actual distance $d$ in the $2 D$-space between the query point and the retrieved data point, and issue a range query centered at the query point with radius $d$. In this way, we need to check all points and return the closest point to the query point. In Figure 5-(a) (Peano curve of order 2), we calculate the sequence number of the query point, i.e., $F_{9}$. We find the proceeding and succeeding sequence numbers on the curve path until one of the points corresponds to $H$. In this case, $H_{10}$ is the first one found to be the nearest neighbor to $F_{9}$. The distance from $H_{10}$ to $F_{9}$ is $2(=d)$ blocks in the $2 D$-space shown in Figure 5 - (a). Then, we check all points which are within two blocks of $F_{9}$ to see if any closer hydrant exists by performing a range query. From Figure 5-(a), we see that a hydrant $H_{3}$ exists which is only one block away from $F_{9}$ in the $2 D$-space. But in the $1 D$-diagram shown in Figure 6-(a), which represents the ordering of the curve, we need to check six points (from 9 to 3) for the range query to obtain the data point $H_{3}$, which is the nearest neighbor (one block away from $F_{9}$ ) in the $2 D$-space. Similarly, in Figure 5-(b) (the RBG curve of order 2), $H_{2}$ is the nearest neighbor, which 


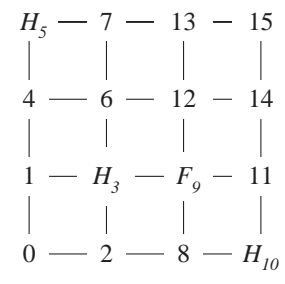

(a)

H: a hydrant

$F$ : the fire

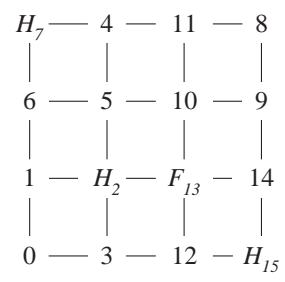

(b)

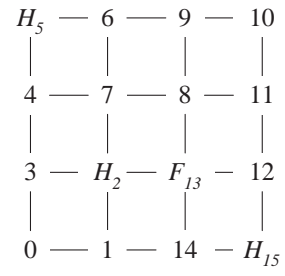

(c)

Figure 5: An example of the nearest neighbor query which is represented by three kinds of ordering: (a) z-ordering; (b) r-ordering; (c) h-ordering.

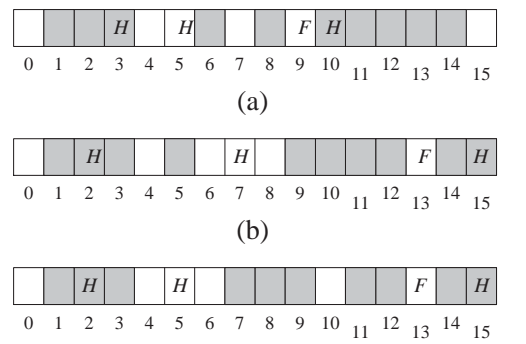

(c)

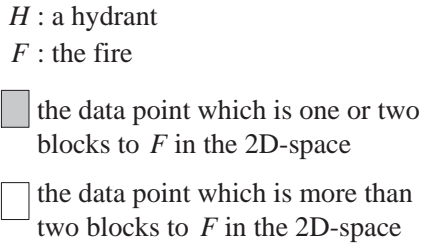

Figure 6: The shaded regions of one-dimensional diagrams illustrate the hydrants $H$ which are within two blocks of the fire $F$ in the $2 D$-space, as shown in Figure 5: (a) z-ordering; (b) r-ordering; (c) h-ordering.

is only one block away from $F_{13}$ in the $2 D$-space. But in the $1 D$-diagram shown in Figure 6-(b), we need to check eleven points (from 13 to 2) for the range query to obtain the data point $\mathrm{H}_{2}$. In Figure 5-(c) (the Hilbert curve of order 2), $\mathrm{H}_{2}$ is the nearest neighbor, which is only one block away from $F_{13}$ in the $2 D$-space. But in the $1 D$-diagram shown in Figure 6-(c), we need to check eleven points (from 13 to 2) for the range query to obtain the data point $\mathrm{H}_{2}$. Thus, when the data is linearly ordered by the space-filling curves, it is time-consuming in the nearest neighbor finding to check all the data in the database by using the conventional approach.

In this section, we first show how to find the nearest neighbor based on the property of the Peano curve. Then, we present the neighbor-finding algorithm based on the RBG curve and the Hilbert curve. We also present the transformation rules between the Peano curve and the other two curves in the neighbor-finding process. 


\subsection{Based on the Peano Curve}

In each of the space-filling curves, the block is characterized by the unique value, i.e., the sequence number. The Peano curve uses the $z$-value to linearly order the blocks. As mentioned in Section 2, the $z$-value in base 2 of the Peano curve can be easily obtained by interleaving the bits from $x_{i}$ bits and then $y_{i}$ bits of two coordinates in base 2. (Note that the value in base 2 is represented with the base 2 bits, i.e., 0 or 1 . A base 2 bit represents that bit times a power of 2.) The relationship between the $z$-value and the coordinates can be shown in Figure 4-(a). The value in parentheses denotes the value in base 2, and the mark (_) denotes the bit obtained from the $X$ coordinate in base 2 . However, it is not so straightforward to obtain the two coordinates in base 2 from the $r$-value of the RBG curve or from the $h$-value of the Hilbert curve in the same way. To obtain the two coordinates in base 2 from the $r$-value requires the reflection recursively on the basic RBG curve of order 1, as shown in Figure 2. To obtain the two coordinates in base 2 from the $h$-value requires the reflection and rotation recursively on the basic Hilbert curve of order 1, as shown in Figure 3. As compared to the $r$-value or the $h$-value, the $z$-value has a direct relationship with the coordinates through the bit shuffling, even when the order is greater than 1. Therefore, in this section, we first describe the process of neighbor-finding based on the $z$-value of the Peano curve.

To simplify our presentation, we let $z 2$ be the $z$-value in base 2 , i.e., the binary form, and $z 10$ be the $z$-value in base 10 , i.e., the decimal form. (Note that the value in base 10 is represented with base 10 digits from 0 to 9 . A base 10 digit represents that digit times a power of 10.) For the query block numbered as $(Z)_{z 10}$, it is the $z$-value of the block in the $2^{n} \times 2^{n}$ image space which is ordered by the Peano curve. The value $(Z)_{z 2}$ is represented in base 2 with $2 n(=n+n)$ bits. So, the sequence numbers for the image space range from 0 to $2^{2 n}-1$. The nearest neighbor algorithm based on the Peano curve is shown in Figure 7.

In Step 1, we separate $(Z)_{z 2}$, e.g., $\left(x_{2} y_{2} x_{1} y_{1} x_{0} y_{0}\right)_{z 2}$, into two parts. One part concatenates the odd bits to the $X$-coordinate, i.e, $X_{c 2}=\left(x_{2} x_{1} x_{0}\right)_{c 2}$, where $c 2$ denotes that the value of the coordinate is represented in base 2. The other part concatenates the even bits to the $Y$-coordinate, i.e., $Y_{c 2}=\left(y_{2} y_{1} y_{0}\right)_{c 2}$. Then, we convert the coordinates $(X, Y)_{c 2}$ 
into $(X, Y)_{c 10}$, where $c 10$ denotes that the value of the coordinate is represented in base 10. In Step 2, we use $(X, Y)_{c 10}$ to find the neighbor in a certain direction. The variables $H D$ and $V D$ mean the horizontal and vertical directions, respectively. For example, if we want to find the west neighbor, we let $H D=$ 'west' and $V D=$ ' $N U L L$ '. Then, we obtain the resulting coordinates $(N X, N Y)_{c 10}$ of the neighbor, e.g., $N X_{c 10}=X_{c 10}-1$ and $N Y_{c 10}=Y_{c 10}$ for the case of the west neighbor. It should be noticed that either $N X_{c 10}$ or $N Y_{c 10}$ of the neighbor must not be beyond the range of the image space in Step 2. If either one of them is beyond the range, there is no neighbor in a certain direction. In Step 3 , we convert $(N X, N Y)_{c 10}$ into $(N X, N Y)_{c 2}$, e.g., $\left(x_{2}^{\prime} x_{1}^{\prime} x_{0}^{\prime}, y_{2}^{\prime} y_{1}^{\prime} y_{0}^{\prime}\right)_{c 2}$. Then, we interleave the bits from $N X_{c 2}$ and $N Y_{c 2}$ to form the corresponding $(N Z)_{z 2}$ for the west neighbor, i.e, $\left(x_{2}^{\prime} y_{2}^{\prime} x_{1}^{\prime} y_{1}^{\prime} x_{0}^{\prime} y_{0}^{\prime}\right)_{z 2}$.

Taking Figure 8-(a) as an example, the width $d$ of each block is 1 . For the query block $(45)_{z 10}=\left(x_{2} y_{2} x_{1} y_{1} x_{0} y_{0}\right)_{z 2}=(\underline{101} 1 \underline{01})_{z 2}$, one of its equal-sized neighbors in the west direction can be obtained by following the steps shown in Figure 9. Let $X\left((45)_{z 10}\right)$ and $Y\left((45)_{z 10}\right)$ denote the $X$-coordinate and the $Y$-coordinate of block $(45)_{z 10}=(\underline{1011} \underline{01})_{z 2}$, respectively. First, we separate $(45)_{z 10}=(\underline{10} \underline{1} \underline{101})_{z 2}$ into $X\left((45)_{z 10}\right)$ and $Y\left((45)_{z 10}\right)$, i.e.,

$$
\begin{aligned}
& X\left((45)_{z 10}\right)=X\left((\underline{10} \underline{1} \underline{1} \underline{0} 1)_{z 2}\right)=X\left(\left(x_{2} y_{2} x_{1} y_{1} x_{0} y_{0}\right)_{z 2}\right)=\left(x_{2} x_{1} x_{0}\right)_{c 2}=(\underline{1} \underline{1} \underline{0})_{c 2}=(6)_{c 10} \\
& Y\left((45)_{z 10}\right)=Y\left((\underline{1} \underline{1} \underline{1} \underline{1} 1)_{z 2}\right)=Y\left(\left(x_{2} y_{2} x_{1} y_{1} x_{0} y_{0}\right)_{z 2}\right)=\left(y_{2} y_{1} y_{0}\right)_{c 2}=\left(\begin{array}{lll}
0 & 1 & 1
\end{array}\right)_{c 2}=(3)_{c 10} .
\end{aligned}
$$

Next, the $N X_{c 10}$ and $N Y_{c 10}$ values of the west neighbor are shown as follows:

$$
\begin{aligned}
& N X_{c 10}=X\left((45)_{z 10}\right)-d=(6)_{c 10}-(1)_{c 10}=(5)_{c 10}, \\
& N Y_{c 10}=Y\left((45)_{z 10}\right)=(3)_{c 10} .
\end{aligned}
$$

There is no change in the $\mathrm{Y}$ coordinate of the west neighbor. Then, we convert $(N X, N Y)_{c 10}=$ $(5,3)_{c 10}$ into $(N X, N Y)_{c 2}=(\underline{1} \underline{0} \underline{1}, 0 \quad 11)_{c 2}=\left(x_{2}^{\prime} x_{1}^{\prime} x_{0}^{\prime}, y_{2}^{\prime} y_{1}^{\prime} y_{0}^{\prime}\right)_{c 2}$. Finally, we interleave the bits from $N X_{c 2}$ and $N Y_{c 2}$ to obtain the $N Z_{z 2}$ of the west neighbor equal to $\left(x_{2}^{\prime} y_{2}^{\prime} x_{1}^{\prime} y_{1}^{\prime} x_{0}^{\prime} y_{0}^{\prime}\right)_{z 2}=(\underline{10} \underline{0} 1 \underline{11})_{z 2}$. Finally, we convert $N Z_{z 2}$ into $N Z_{z 10}$ to obtain the west neighbor, and the resulting block $(39)_{z 10}$ is shown in Figure 8-(a). Similarly, the neighbors in the other directions can also be obtained by function Peano_NN, as shown in Figure 7. 
Function Peano_NN $\left((Z)_{z 2}, H D, V D\right)$ : string;

$/ *$ Find the nearest neighbor next to block $(Z)_{z 2}$ based on the Peano curve in the certain direction. */

$/ * l_{0}$ and $h_{0}$ are the lower and upper bounds of coordinates along the $X$-axis. */

${ }^{*} l_{1}$ and $h_{1}$ are the lower and upper bounds of coordinates along the $Y$-axis. */

$/ * Z_{z 2}$ is the $z$-value (in base 2 ) of the query block. */

* $H D$ and $V D$ are the horizontal and vertical directions. */

$/^{*}(X, Y)_{c 2}$ is the coordinates (in base 2) of the query block. */

$/^{*}(X, Y)_{c 10}$ is the coordinates (in base 10) of the query block. */

/* $(N X, N Y)_{c 10}$ is the coordinates (in base 10) of the resulting neighboring block. */

${ }^{*}(N X, N Y)_{c 2}$ is the coordinates (in base 2) of the resulting neighboring block. */

$/^{*}(N Z)_{z 2}$ is the $z$-value (in base 2 ) of the resulting neighboring block. */

begin

/* Step 1: Bit Separation */

Separate the binary code of $Z_{z 2}$ into the odd bits and the even bits;

Concatenate the odd bits to $X_{c 2}$;

Concatenate the even bits to $Y_{c 2}$;

Convert $X_{c 2}$ into $X_{c 10}$;

Convert $Y_{c 2}$ into $Y_{c 10}$;

/* Step 2: Neighbor Decision */

if $(H D=$ 'west')

then $X_{c 10}:=X_{c 10}-1$

else if $(H D=$ 'east')

then $X_{c 10}:=X_{c 10}+1$

if $(V D=$ 'south')

then $Y_{c 10}:=Y_{c 10}-1$

else if $(V D=$ 'north')

then $Y_{c 10}:=Y_{c 10}+1$

if $\left(X_{c 10}<l_{0}\right)$ or $\left(X_{c 10}>h_{0}\right)$ or $\left(Y_{c 10}<l_{1}\right)$ or $\left(Y_{c 10}>h_{1}\right)$ then begin

show an error message; /* There exists no neighbor. */

end

$\operatorname{Return}\left({ }^{6}\right.$ ');

else begin

$N X_{c 10}:=X_{c 10}$

end;

$N Y_{c 10}:=Y_{c 10}$

/* Step 3: Bit Shuffling */

Convert $N X_{c 10}$ into $N X_{c 2}$;

Convert $N Y_{c 10}$ into $N Y_{c 2}$;

Interleave the bits from $N X_{c 2}$ and $N Y_{c 2}$ to form $(N Z)_{z 2}$;

Return $\left((N Z)_{z 2}\right)$; end;

Figure 7: Function Peano_NN 


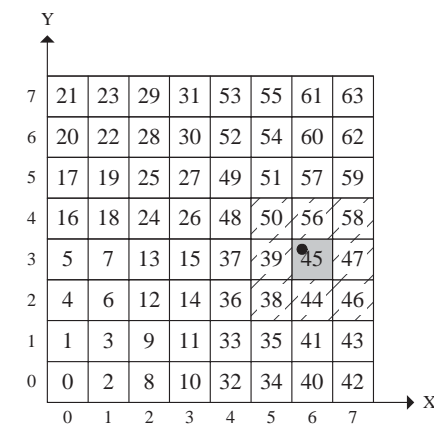

(a)

\begin{tabular}{|c|c|c|c|c|c|c|c|}
\hline 31 & 28 & 19 & 16 & 47 & 44 & 35 & 32 \\
\hline 30 & 29 & 18 & 17 & 46 & 45 & 34 & 33 \\
\hline 25 & 26 & 21 & 22 & 41 & 42 & 37 & 38 \\
\hline 24 & 27 & 20 & 23 & 40 & 43 & 36 & 39 \\
\hline 7 & 4 & 11 & 8 & 55 & 52 & 59 & 56 \\
\hline 6 & 5 & 10 & 9 & 54 & 53 & 58 & 57 \\
\hline 1 & 2 & 13 & 14 & 49 & 50 & 61 & 62 \\
\hline 0 & 3 & 12 & 15 & 48 & 51 & 60 & 63 \\
\hline
\end{tabular}

(b)

\begin{tabular}{|c|c|c|c|c|c|c|c|}
\hline 21 & 22 & 25 & 26 & 37 & 38 & 41 & 42 \\
\hline 20 & 23 & 24 & 27 & 36 & 39 & 40 & 43 \\
\hline 19 & 18 & 29 & 28 & 35 & 34 & 45 & 44 \\
\hline 16 & 17 & 30 & 31 & 32 & 33 & 46 & 47 \\
\hline 15 & 12 & 11 & 10 & 53 & 52 & 51 & 48 \\
\hline 14 & 13 & 8 & 9 & 54 & 55 & 50 & 49 \\
\hline 1 & 2 & 7 & 6 & 57 & 56 & 61 & 62 \\
\hline 0 & 3 & 4 & 5 & 58 & 59 & 60 & 63 \\
\hline
\end{tabular}

(c)

- the query point

block where the query point is located

neighboring block to the query block

Figure 8: The sequence numbers linearly ordered by the curves of order 3: (a) the Peano curve; (b) the RBG curve; (c) the Hilbert curve.

$(45)_{z 10}$

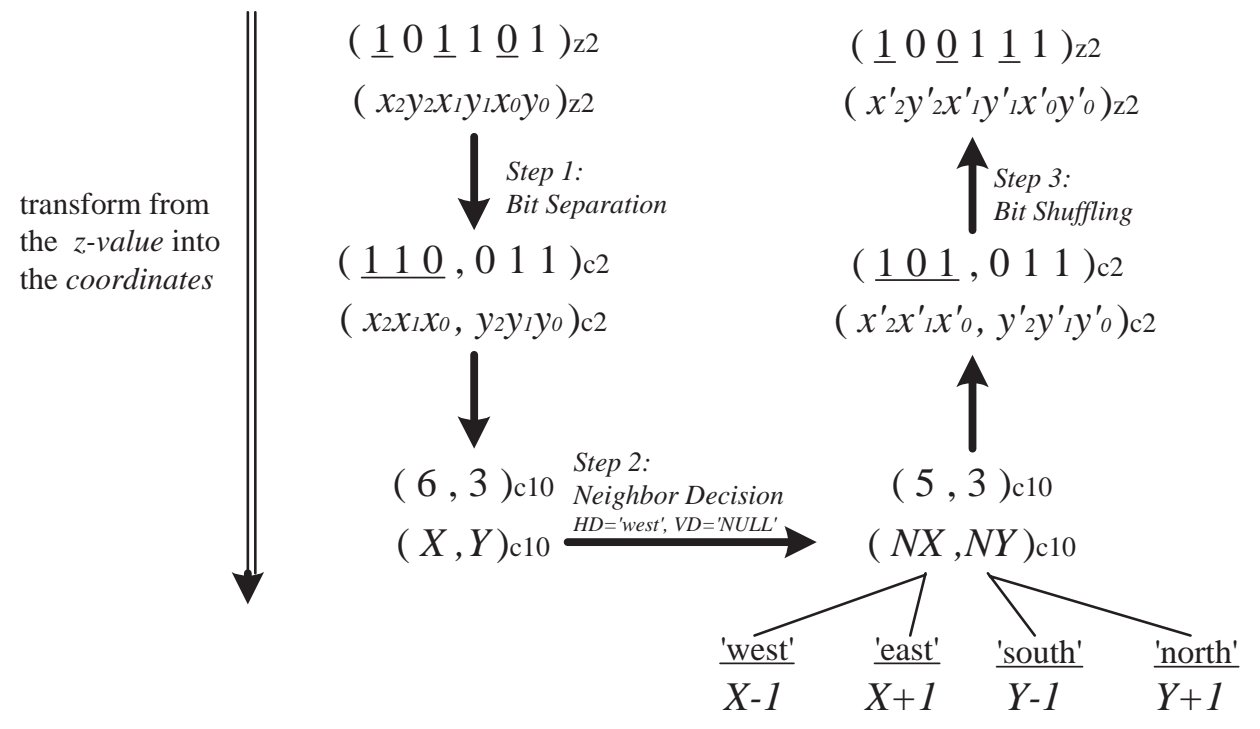

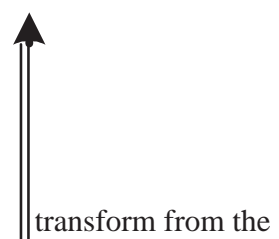
coordinates back into the z-value

Figure 9: Illustration of the west neighbor-finding process for the query block $(45)_{z 10}=$ $(101101)_{z 2}$ 


\subsection{Neighboring-Finding Based on the Other Two Curves}

Based on the Peano curve, we can efficiently and directly find the neighbors next to the query block by function Peano_NN (Figure 7). But it is not so straightforward to answer the nearest neighbor queries based on the RBG curve or the Hilbert curve, as mentioned above. However, we observe that there is some relationship between the RBG curve and the Peano curve, and between the Hilbert curve and the Peano curve. The $r$-value in the RBG curve can be represented as the binary gray-code $(R)_{r 2}$ (also called gray code), where $r 2$ denotes the $r$-value in base 2 . The formulas presented in $[2,3,15]$ can transform $(R)_{r 2}$ into its order in the binary form as $(z)_{z 2}$. The $h$-value in the Hilbert curve can be obtained by some transformation, reflection, and rotation on the $z$-value. So, for the RBG curve (or the Hilbert curve), we can develop some transformation rules between the Peano curve and itself, and use function Peano_NN to help answer the nearest neighbor query. Therefore, we can first transform the $r$-value (or $h$-value) of the query block into the $z$-value. Then, we use the corresponding $z$-value to find the neighbors based on the Peano curve. We finally transform the $z$-value of the neighbor back into the corresponding $r$-value (or $h$-value) of the neighbors based on the transformation rules. In this section, we first briefly describe the process of the neighbor-finding based on the RBG curve. Then, we show how to find the nearest neighbor based on the Hilbert curve.

\subsubsection{Based on the RBG Curve}

As mentioned before, for the $r$-value of the $2 \mathrm{D}$-space RBG curve in the $2^{n} \times 2^{n}$ image space, we can consider the value $(R)_{r 2}$ as the gray code, where $r 2$ denotes the $r$-value in base 2 . That is the bit string $\left(R_{2 n-1} \ldots R_{1} R_{0}\right)_{r 2}$. The neighbor-finding algorithm based on the RBG curve is shown in Figure 10. Basically, we first transform $(R)_{r 2}$ into the corresponding $z$-value, i.e., $(Z)_{z 2}$, by the formulas presented in $[2,3,15]$. That is, we perform the transformation between the gray code and the binary code. Then, we use $(Z)_{z 2}$ to generate one of the neighbors in a certain direction by function Peano_NN. Finally, we transform $(N Z)_{z 2}$ of the neighbor back into the corresponding $(N R)_{r 2}$ by again using the formulas presented in $[2,3,15]$.

Take Figure 8 -(b) as an example. It is a $2^{3} \times 2^{3}$ image space which is ordered by the 
Function $R B G_{-} N N\left((R)_{r 2}, H D, V D\right)$ : string;

${ }^{*}$ Find the nearest neighbor next to block $(R)_{r 2}$ based on the RBG curve in a certain direction. */

$/^{*}(R)_{r 2}$ is the gray code of length $2 n$-bits for the query block in the RBG curve. */

$/ *(Z)_{z 2}$ is the $z$-value in base 2 of length $2 n$-bits for the query block in the Peano curve. */

${ }^{*}(N R)_{r 2}$ is the gray code of the neighboring block in the RBG curve. */

${ }^{*}(N Z)_{z 2}$ is the $z$-value in base 2 of the neighboring block in the Peano curve. */

begin

/* Step 1: Transformation from Gray Code into Binary Code*/

/* In other words, to transform the RBG curve into the Peano curve*/

$/ * \bigoplus$ is the exclusive-or operator. */

the left significant bit of $(Z)_{z 2}:=$ the left significant bit of $(R)_{r 2}$;

for the following bits of $(Z)_{z 2}$

$$
(Z)_{z 2(2 n-1-i)}:=(R)_{r 2(2 n-1-i)} \bigoplus(Z)_{z 2(2 n-i)} ;
$$

/* Step 2: Neighbor Decision */

$(N Z)_{z 2}:=$ Peano_NN $\left((Z)_{z 2}, H D, V D\right)$;

/* Step 3: Transformation from Binary Code into Gray Code*/

/* In other words, to transform the Peano curve into the RBG curve*/

the left significant bit of $(N R)_{r 2}:=$ the left significant bit of $(N Z)_{z 2}$;

for the following bits of $(N R)_{r 2}$

$(N R)_{r 2(2 n-1-i)}:=(N Z)_{z 2(2 n-1-i)} \bigoplus(N Z)_{z 2(2 n-i)} ;$

Return $\left((N R)_{r 2}\right)$;

end;

Figure 10: Function $R B G_{-} N N$ 


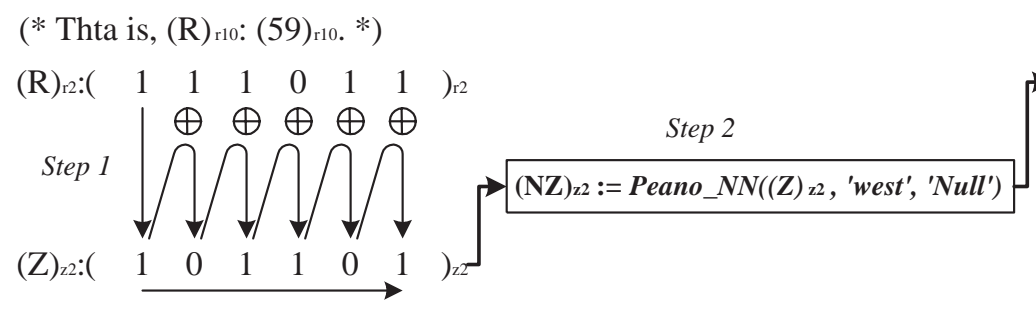

(* That is, $\left.(\mathrm{Z})_{\mathrm{z} 10}:(45)_{\mathrm{z} 10 .} *\right)$

$$
\text { (* That is, } \left.(\mathrm{NZ})_{\mathrm{z} 10}:(39)_{\mathrm{z} 10} *{ }^{*}\right)
$$

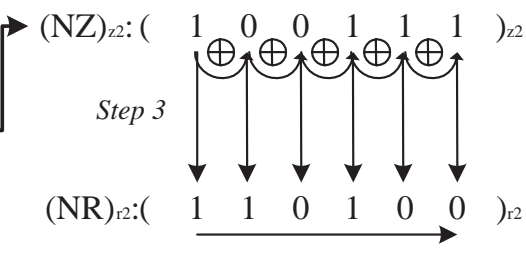

(* That is, $(\mathrm{NR})_{\mathrm{r} 10}:(52)_{\mathrm{r} 10} *$ )

Figure 11: Illustration of the west neighbor-finding process for the query block $(59)_{r 10}=(111011)_{r 2}$

RBG curve of order 3 . The query point is located in block $(59)_{r 10}$, where $r 10$ denotes the $r$-value in base 10 . We use block $(59)_{r 10}$ as the query block which has the same coordinates $(X, Y)_{c 10}=(6,3)_{c 10}$ as the example shown in Figure 8-(a). We represent the r-value $(59)_{r 10}$ in base 2 as the gray code, i.e., $\left(R_{5} R_{4} R_{3} R_{2} R_{1} R_{0}\right)_{r 2}=(111011)_{r 2}$. The west neighbor finding process of block $(59)_{r 10}$ is shown in Figure 11. In Step 1, we transform the gray code $(R)_{r 2}=(111011)_{r 2}$ into the $z$-value in base 2, i.e., $(Z)_{z 2}=(\underline{101} 1 \underline{01})_{z 2}$. In Step 2, we use the $z$-value $(\underline{10} \underline{1} 1 \underline{0} 1)_{z 2}=(45)_{z 10}$ to find the west neighbor by function Peano_NN. Then, we find the $z$-value $(\underline{10} \underline{0} 1 \underline{11})_{z 2}=(39)_{z 10}$ of the neighboring block. In Step 3, we transform the $z$-value $(\underline{1} 0 \underline{0} 1 \underline{11})_{z 2}$ back into the $r$-value $(110100)_{r 2}$. Finally, we convert the $r$-value $(110100)_{r 2}$ into the decimal $(52)_{r 10}$ which is the neighbor lies the west of the query block $(59)_{r 10}$ in Figure 8-(b). Note that block $(52)_{r 10}$ also locates at $(N X, N Y)_{c 10}=(5,3)_{c 10}$, so we have the same answer as the previous example shown in Figure 8-(a).

\subsubsection{Based on the Hilbert Curve}

For the $h$-value of the $2 D$-space Hilbert curve in the $2^{n} \times 2^{n}$ image space, we consider the value $H$ in base 2 as a Hilbert code, i.e., $\left(h_{2 n-1} \ldots h_{1} h_{0}\right)_{h 2}$, where $h 2$ denotes the $h$-value in base 2. The pseudo codes for the neighbor-finding process is shown in Figure 12, and the related source codes about the transformation rules between the Peano curve and the Hilbert curve are shown in Appendix A. Basically, similar to the previous function, from Step 1 to Step 4, we first transform the $h$-value into the $z$-value. Then, in Step 5 , we use the $z$-value to generate the requested neighbor based on the Peano curve. Finally, from 
Step 6 to Step 9, we transform the $z$-value of the neighbor into the corresponding $h$-value. However, in this case, it is not so straightforward to do the transformation between the Hilbert curve and the Peano curve since the coordinates in base 2 from the $h$-value require the reflection and rotation recursively on the basic Hilbert curve of order 1 .

Take Figure 8-(c) as an example. It is a $2^{3} \times 2^{3}$ image space which is ordered by the Hilbert curve of order 3 . The query point is located in block $(51)_{h 10}$, where $h 10$ denotes the $h$-value in base 10 . We use block $(51)_{h 10}$ as the query block, which has the same coordinates $(X, Y)_{c 10}=(6,3)_{c 10}$ as the example shown in Figure 8-(a). The west neighborfinding process of block $(51)_{h 10}$ is shown in Figure 13. We first represent the $h$-value, i.e., $(51)_{h 10}$, in base 2 as the Hilbert code $\left(h_{5} h_{4} h_{3} h_{2} h_{1} h_{0}\right)_{h 2}=(110011)_{h 2}$. We describe the neighbor-finding process of block $(51)_{h 10}$ in the following steps.

Step 1: We divide the Hilbert code $(\underline{110011})_{h 2}$ into pairs of bits from left to right and store the corresponding decimal value for each pair of bits in array $d$. Those are $d_{0}=(\underline{11})_{2}=3, d_{1}=(00)_{2}=0$, and $d_{2}=(11)_{2}=3$. The decimal value denotes the sequence number $(0,1,2$, or 3$)$ in one quadrant ordered by the Hilbert curve, as shown in Figure 3-(a). We also let another array $e$ be same as array $d$, i.e., $e_{0}=d_{0}=3$, $e_{1}=d_{1}=0, e_{2}=d_{2}=3$. (Note that array $e$ is used for storing the temporary result in the following steps.) Array $d$ is represented as $\left(d_{0} d_{1} d_{2}\right)_{h p}=\left(\begin{array}{lll}3 & 0 & 3\end{array}\right)_{h p}$, where $h p$ denotes the code composed of the sequence numbers in base 10 for pairs of bits in $(H)_{h 2}$, and so is array e. Note that since all reflections and rotations in the Hilbert curve of order $p, p>1$ are relative to the previously obtained curve of order $p-1$, array $d$ is the input used to store the sense of order $p$, and array $e$ is the output used to store the sense of order $p-1$. In other words, for the following step, there will be no change to array $d$ to keep the information about reflection and rotation in the curve of order $p$. And array $e$ will be repeatedly updated (if necessary) to show the sense in the curve of order $p-1$.

Step 2: We call procedure Rotate_Reflect, as shown in Figure 14, to perform the task of reducing the Hilbert curve from order $i(i>1)$ to order 1. For each element in array $d$, we first consider the value of $d_{i}$ to decide which case to follow in procedure Rotate_Reflect, as shown in Figure 14. Arrays $d$ and $e$ are the input and output, 
Function $H i l b e r t \_N N\left((H)_{h 2}, H D, V D\right)$ : string;

$/^{*}$ Find the nearest neighbor next to block $(H)_{h 2}$ based on the Hilbert curve in the certain direction. */

$/ *(H)_{h 2}$ is the Hilbert code of the query block. */

$/^{*}(Z)_{z 2}$ is the $z$-value in base 2 of the query block. */

$/^{*}(N H)_{h 2}$ is the Hilbert code of the neighboring block. */

${ }^{*}(N Z)_{z 2}$ is the $z$-value in base 2 of the neighboring block. */

$/ * c, d, e$ are arrays to store the decimal values. */

begin

/* Step 1: Bit Division */

for each pair $i$ of bits in $(H)_{h 2}$ from left to right

begin

Convert them into the decimal value $d_{i}$

$e_{i}:=d_{i}$

end;

/* Step 2: Reducing the Hilbert curve from order $i$ to order $1, i>1$ */

Call Rotate_Reflect $(d, e)$;

/* Step 3: Transformation from the Hilbert Curve into the Peano Curve */

Call PHTransformation(e);

/* Step 4: Bit Concatenation */

for each $e_{i}$ in $e$

Concatenate the binary bits of $e_{i}$ to $(Z)_{z 2}$;

/* Step 5: Neighbor Decision based on the Peano curve */

$(N Z)_{z 2}:=$ Peano_NN $\left((Z)_{z 2}, H D, V D\right)$;

/* Step 6: Bit Division */

for each pair $i$ of bits in $(N Z)_{z 2}$ from left to right

Convert them into the decimal value $c_{i}$;

/* Step 7: Transformation from the Peano Curve into the Hilbert Curve*/

Call PHTransformation $(c)$;

/* Step 8: Generating the Hilbert curve of order $i$ from order $1, i>1 * /$

Call Rotate_Reflect $(c, c)$;

/* Step 9: Bit Concatenation */

for each $c_{i}$ in $c$

Concatenate the binary bits of $c_{i}$ to $(N H)_{h 2}$;

Return $\left((N H)_{h 2}\right)$;

end;

Figure 12: Function Hilbert_NN 


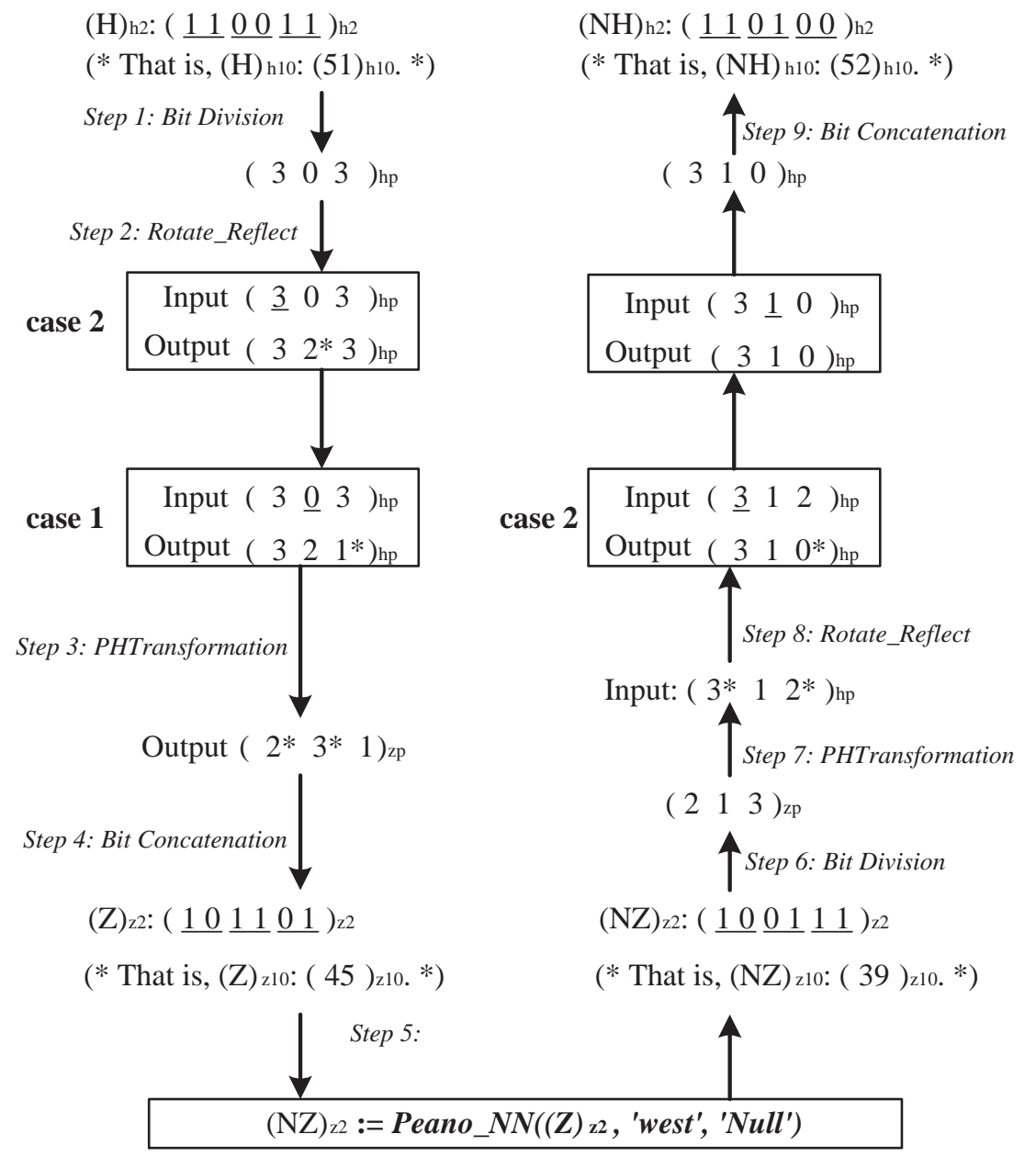

Figure 13: Illustration of the west neighbor-finding process for the query block $(51)_{h 10}=$ $(110011)_{h 2}$ 
Procedure Rotate_Reflect(input, output);

/* Transformation between the Hilbert curve of order $p$ and order $q * /$

/* input is an array to store the sequence numbers for the curve of order p. */

${ }^{*}$ output is an array to store the sequence numbers for the curve of order q. */

begin

for $i=1$ to $\mid$ input $\mid-1$ do

begin

/* Case $1 * /$

if $($ input $[i]=0)$ then

for the following element $j:=i+1$ to $\mid$ output $\mid$ do

begin

if $($ output $[j]=1)$ then output $[j]:=3$

else if (output $[j]=3$ ) then output $[j]:=1$;

end;

/* Case $2^{*} /$

else if (input $[i]=3$ ) then

for the following element $j:=i+1$ to $\mid$ output $\mid$ do

begin

if $($ output $[j]=0)$ then output $[j]:=2$

else if (output $[j]=2)$ then output $[j]:=0$; end;

end;

end;

Figure 14: Procedure Rotate_Reflect

respectively. In Case 1 , if the value of $d_{i}$ is 0 , then we should change the value of the following element $e_{j}$ from 1 to 3 , or from 3 to $1, j>i$. In Case 2 , if the value of $d_{i}$ is 3 , then we should change the value of the following element $e_{j}$ if the value is from 0 to 2 , or from 2 to $0, j>i$. In Figure 13, the input is array $d=\left(\begin{array}{lll}3 & 0 & 3\end{array}\right)_{h p}$ and the output is array $e=\left(\begin{array}{lll}3 & 0 & 3\end{array}\right)_{h p}$ initially, where the mark _ represents the processing point. First, we process the first value of the input, i.e., $d_{0}(=3)$. Since it is 3 , which is Case 2, we change the second value of the output $\left(e_{1}\right)$ from 0 to 2 . Since there is no other 0 or 2 in the remaining $e_{j}, j>i$, we do not do any other update to the output for the input $d_{0}$. (Note that in Figure 13, the mark * represents the changed value.) Next, we process the second value of the input, i.e., $d_{1}(=0)$. Since it is 0 , which is Case 1, we change the third value of the output $\left(e_{2}\right)$ from 3 to 1 . Up to this point, we have finished the process of all elements of the input, and the resulting output is (3 $\left.2 \begin{array}{lll}3 & 1\end{array}\right)_{h p}$.

The reason for the change operation for Case 1 and Case 2 is as follows. In order 
to preserve the proximity, the order $p(p>1)$ of the Hilbert curve is derived from the rotation and reflection on the basic curve of order 1 continuously in each quadrant. The sense, i.e., the direction of traversal [6], in each quadrant is not always the same. However, different from the Hilbert curve, the Peano curve recursively shows the same sense as the basic curve of order 1 to form the curve of order $p, p>1$. So, during the process of the transformation between the $h$-value and the $z$-value, we need to perform the rotation and reflection in reverse order such that each divided quadrant will have the same sense as the basic Hilbert curve of order 1 .

Take Figure 15 as an example. Figures 15-(a) and (b) show the Hilbert curves of order 1 and 2, respectively. The ( ) denotes the sequence number of the quadrant in the Hilbert curve of order 1, in which the quadrant has four blocks. Each block has the sequence number ordered by the Hilbert curve of order 2 . We can observe that the sense of block (0) of order 2 in Figure 15-(b) is different from the sense of order 1 in Figure 15-(a), as is block (3). That is, in block (0), the position of sequence number 1 is different from its position in Figure 15-(a), as is the position of sequence number 3. It is just the sense of block (0) of order 2 (Figure 15-(b)) as derived from the the sense of one of order 1 (Figure 15-(a)) by right-reflection (Figure 16-(a)) and 90-clockwiserotation (Figure 16-(b)). After processing the reflection and rotation, the result is shown in Figure 16-(c). This is Case 1 in procedure Rotate_Reflect. Similarly, for block (3) in Figure 15-(b), the position of sequence number $12(=4 \times 3+\underline{0})$ is different from its position (sequence number $\underline{0}$ ) in Figure 15-(a), so is the position of sequence number $14(=4 \times 3+\underline{2})$ vs. the position of sequence number 2 . This is because the sense of block (3) (Figure 15-(b)) is derived from the the sense of order 1 (Figure 15(a)) by left-reflection (Figure 17-(a)) and 90-anticlockwise-rotation (Figure 17-(b)). After processing the reflection and rotation, the result is shown in Figure 17-(c). This is Case 2 in procedure Rotate_Reflect. However, the sense of block (1) in Figure 15-(b) is the same as that of order 1 (Figure 15-(a)) without processing rotation and reflection, so is block (2). Therefore, to reduce the Hilbert curve of order $p(p>1)$ to the basic curve of order 1, we have to perform procedure Rotate_Reflect. In this way, after all senses in the Hilbert curve of order $p(p>1)$ have been reduced to the ones 


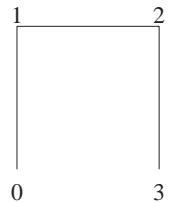

(a)

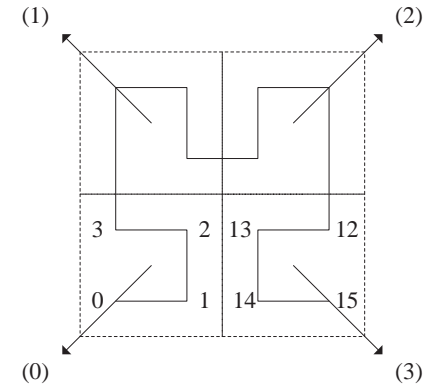

(b)

$\left(N_{1}\right): N_{1}$ is the sequence number of the quadrant in the curve of order 1.

Figure 15: Hilbert curves of order: (a) 1; (b) 2.

in the basic Hilbert curve of order 1, we can do the transformation from the Hilbert curve into the Peano curve.

Step 3: Up to this point, we have the code of $\left(\begin{array}{lll}3 & 2 & 1\end{array}\right)_{h p}$, which is the code of the Hilbert curve of order 1. Note that in procedure PHTransformation, as shown in Figure 18, we observe that the position of sequence number 2 in the basic Hilbert curve of order 1 (Figure 19-(a)) is different from its position in the basic Peano curve of order 1 (Figure 19-(b)), as is sequence number 3. Therefore, we change the position of sequence numbers 2 and 3 in the basic Hilbert curve of order 1 to those in the basic Peano curve to obtain the $z$-value. So, we transform $\left(\begin{array}{lll}3 & 2 & 1\end{array}\right)_{h p}$ into the corresponding code of the $z$-value, i.e., $\left(2^{*} 3^{*} 1\right)_{z p}$, according to Table 1 by procedure PHTransformation, where $z p$ denotes the code composed of sequence numbers in base 10 for pairs of bits in $(Z)_{z 2}$.

Step 4: We transform each number of $(\underline{2} \underline{3} \underline{1})_{z p}$ into the corresponding pair of bits. Then, we concatenate them to form $(\underline{10} \underline{11} \underline{01})_{z 2}$.

Step 5: We use the $z$-value $(101101)_{z 2}=(45)_{z 10}$ to find the nearest neighbor to the west based on the Peano curve by function Peano_NN. We find the west neighbor which is $(100111)_{z 2}=(39)_{z 10}$ in Figure 8-(a). 


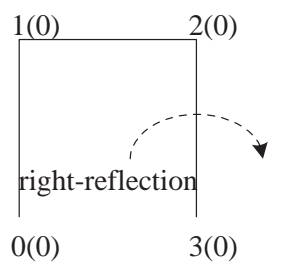

(a)

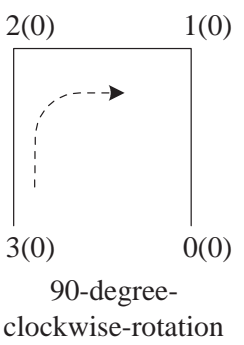

(b)

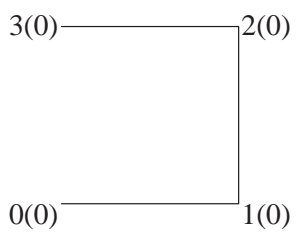

(c)

$N_{2}\left(N_{1}\right): N_{1}$ is the sequence number of the block in the curve of order 1 , and $N_{2}$ is the sequence number of the block in the curve of order 2 .

Figure 16: The process of reflection and rotation on block (0) in the Hilbert curve of order 2 in Figure 15: (a) before the right reflection; (b) after the right reflection; (c) after the $90^{\circ}$-clockwise-rotation.

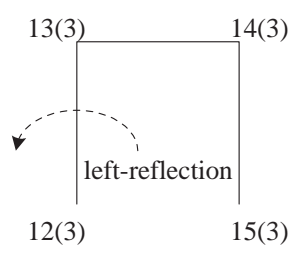

(a)

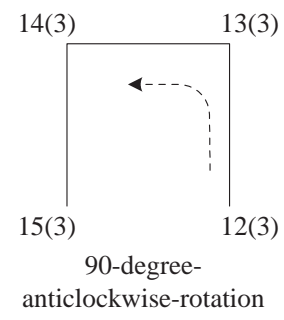

(b)

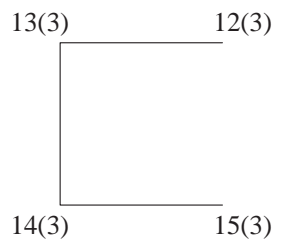

(c)

$N_{2}\left(N_{1}\right): N_{1}$ is the sequence number of the block in the curve of order 1 , and $N_{2}$ is the sequence number of the block in the curve of order 2 .

Figure 17: The process of reflection and rotation on block (3) in the Hilbert curve of order 2 in Figure 15: (a) before the left reflection; (b) after the left reflection; (c) after the $90^{\circ}$-anticlockwise-rotation.

Table 1: The relationship between the sequence numbers in the Peano curve and those in the Hilbert curve

\begin{tabular}{|c|c|}
\hline Peano curve & Hilbert curve \\
\hline 0 & 0 \\
1 & 1 \\
2 & 3 \\
3 & 2 \\
\hline
\end{tabular}


Procedure PHTransformation $(\mathrm{m})$;

/* Transformation between the Peano curve and the Hilbert curve * /

${ }^{*} m$ is an array to store the decimal values. */

begin

for each $i:=1$ to $|m|$ do

begin

if $(m[i]=2)$ then $m[i]:=3$

end;

else if $(m[i]=3)$ then $m[i]:=2$;

end;

Figure 18: Procedure PHTransformation

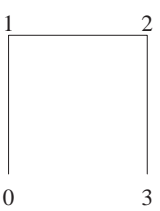

(a)

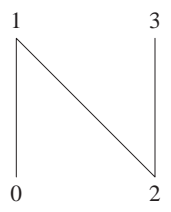

(b)

Figure 19: Curves of order 1: (a) the Hilbert curve; (b) the Peano curve.

Step 6: We divide the code $(\underline{100111})_{z 2}$ into pairs of bits from left to right and store the corresponding decimal value for each pair of bits in array $c$. These are $c_{0}=(\underline{10})_{2}=2$, $c_{1}=(01)_{2}=1$, and $c_{2}(11)=3$. We represent array $c$ as $\left(\begin{array}{lll}2 & 1 & 3\end{array}\right)_{z p}$, which represents the code of sequence numbers in the Peano curve.

Step 7: In this step, based on the similar reason as Step 3, we change the position of sequence numbers 2 and 3 in the basic Peano curve of order 1 to those in the basic Hilbert curve of order 1 . We transform the code of $\left(c_{0} c_{1} c_{2}\right)_{z p}=\left(\begin{array}{lll}2 & 1 & 3\end{array}\right)_{z p}$ into the corresponding $h p$ code in the basic Hilbert curve of order 1 according to Table 1 by using procedure PHTransformation in Figure 18. At this point, we have the resulting array $c$ as $\left(3^{*} 12^{*}\right)_{h p}$, which represents the code of sequence numbers in the basic Hilbert curve of order 1.

Step 8: In this step, with a reason similar to Step 2, we should generate the Hilbert curve of order $p(p>1)$ from the basic Hilbert curve of order 1. For each element in array $c$, we consider the value of $c_{i}$ to decide which case to follow in procedure Rotate_Reflect (Figure 14). Note that since all reflections and rotations in the Hilbert curve of order 
$p, p>1$ are relative to the previously obtained curve of order $p-1$, the array $c$ is not only the input, which represents the sense of order $p-1$, but also the output, which represents the sense of order $p$. First, we process the first value of the input, i.e., $c_{0}$ $(=3)$. Since it is 3 , which is Case 2 , we change only the third value of the output $\left(c_{2}\right)$ from 2 to 0 . Since there is no other 0 or 2 in the second value $c_{1}(=1)$ and the remaining $c_{j}, j>i$, we do not do any other update to the output for the input $c_{0}$. Next, we process the second value of the input, i.e., $c_{1}(=1)$. Since it is 1 , we do not update the output. Up to this point, we have finished the process of all elements of the input, and the resulting output is $\left(\begin{array}{lll}3 & 1 & 0\end{array}\right)_{h p}$, which represents the code of sequence numbers in the Hilbert curve of order $p(p>1)$.

Step 9: We transform each number of $\left(c_{0} c_{1} c_{2}\right)_{h p}=\left(\begin{array}{lll}3 & 1 & 0\end{array}\right)_{h p}$ into the corresponding pair of bits. Then, we concatenate them to form $(\underline{11} \underline{01} \underline{00})_{h 2}$. Finally, we obtain block $(52)_{h 10}=(110100)_{h 2}$ as the west neighbor next to block $(51)_{h 10}$ as shown in Figure 8-(c). Note that block $(52)_{r 10}$ also locates at $(N X, N Y)_{c 10}=(5,3)_{c 10}$, which is the same answer as the previous example in Figure 8-(a).

\section{Performance}

In this section, we compare the performance of nearest neighbor-finding strategies based on three space filling curves. Our experiments were performed on a Pentium III $733 \mathrm{MHz}$, 128 MB RAM, 7200 rpm IBM Hard Disk (model name: IC35L040AVVN07-0), and running Windows 2000.

\subsection{The Performance Model}

In the conventional approach as mentioned in Section 3, it requires the time to traverse the disk blocks by following the sequence numbers in the preceding and succeeding order of the query block in the $1 D$-space to find the neighboring blocks. The number of these traversed neighboring blocks are unpredictable and may be greater than 8 blocks, since some unrelated disk blocks in the $1 D$-space may be accessed. Take the query block 13 in Figure 6-(c) based on the Hilbert curve as an example, it will access 11 blocks in the 
$1 D$-space to find one neighboring block 2, which is one block away from the query block 13 in the $2 D$-space. As compared to the conventional approach, our approach does not need to access unrelated disk blocks in the $1 D$-space. In our approach, first, we compute the sequence numbers of the neighboring blocks next to the query block in 8 directions. It takes the CPU-time to compute these sequence numbers based on the space-filling curve. Next, once the sequence numbers of the 8 neighboring blocks are obtained, we can fetch the corresponding blocks in the disk directly according to the sequence numbers. Then, we can find the nearest point to the query point only in these 8 neighboring blocks in the $2 D$-space, which speeds up the search operation.

The I/O time, which means the time to access a block, consists of two parts: positioning time (PT) and transfer time (TT) [17]. The positioning time is the time to move to the right position in the disk, which includes the seek time and rotational delays. The transfer time is simply the time to transfer the requested block from the disk into main memory. Since it is more efficient to fetch a set of consecutive disk blocks than a randomly scattered set, in order to reduce additional positioning time, we can make use of the clustering property of the space filling curve to replace several exact match queries with one continuous range query to reduce the positioning time. The clustering property means that the locality between objects in the $2 D$-space can be preserved in the $1 D$-space. In our approach, since the adjacent blocks on the disk can be accessed with a single range query, we can combine 8 exact match queries into some range queries to reduce the positioning time.

Take Figure 8 as an example. In the case of the Peano curve as shown in Figure 8-(a), let's let the query block be block 45 . The sequence numbers of the neighboring blocks in 8 directions are blocks 56, 58, 47, 46, 44, 38, 39, and 50. Then, we can combine these 8 exact match queries into 6 range queries, including $\underline{38 \text { to } 39}, \underline{44}, \underline{46 \text { to } 47}, \underline{50}, \underline{56}$, and $\underline{58}$. The positioning time for 6 range queries is $6 * P T$. The transfer time to transfer these 8 blocks from the disk is $8 * T T$. So, the total I/O time to access these 8 disk blocks based on the Peano curve is $(6 * P T+8 * T T)$. (Note that in this case, the number of blocks which the conventional approach has to access, is up to 21 (from 38 to 58) blocks in the worst case. Moreover, in the conventional approach, the higher the order of the space-filling curve is, the large the number of blocks accessed $[4,9,11,12]$. 
In the case of the RBG curve as shown in Figure 8-(b), let's let query block be block 59. The sequence numbers of the neighboring blocks in 8 directions are blocks 36, 39, 56, 57, $58,53,52$, and 43 . Then, we can combine these 8 exact match queries into 5 range queries,

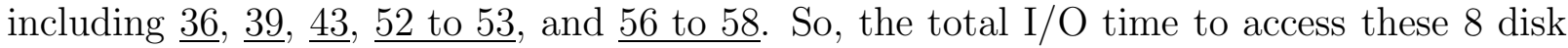
blocks based on the RBG curve is $(5 * P T+8 * T T)$.

In the case of the Hilbert curve as shown in Figure 8-(c), let's let the query block be block 51. The sequence numbers of the neighboring blocks in 8 directions are blocks 46 , $47,48,49,50,52,55$, and 33 . Then, we can combine these 8 exact match queries into 4 range queries, including $\underline{33}, \underline{46 \text { to } 50}, \underline{52}$, and $\underline{55}$. So, the total I/O time to access these 8 disk blocks based on the Hilbert curve is $(4 * P T+8 * T T)$. Therefore, as compared to the conventional approach, our approach can reduce the I/O time in accessing the unrelated blocks by directly accessing the 8 related blocks. Moreover, in this example, among three space-filling curves, our strategy based on the Hilbert curve needs the least I/O time because the Hilbert curve provides the best clustering property $[4,6,7,8,12]$.

In this comparison, we considered the CPU-time and the I/O time as our performance measures. We focused on the point data. The experimental analysis was carried out on six distinct spatial datasets. Figure 20 shows these six cases of data distribution which are described as follows [16]:

(a) Uniform Distribution: The data objects are uniformly distributed in the overall data space.

(b) Centralized Distribution: Most of the data objects are centralized in a small region.

(c) Diagonal Distribution: The data objects follow a uniform distribution along the main diagonal.

(d) X-parallel Distribution: Most of the data objects are located on a line which is parallel to the $\mathrm{X}$-axis.

(e) Sine Distribution: The data objects follow a sine curve.

(f) Real map: This is the point map of the population estimate in Taipei, Taiwan. There are 26376 data points on this map. One point represents 100 people. This statistical 
information is retrieved from http://www.taiwandm.com.tw/c1-1.htm.

Each dataset contains 10000 data points in the $2 D$-space which is $1000 \times 1000$. First, the data points were assigned to the corresponding disk block based on the space-filling curve. The points in the same disk block are linked. The capacity of each disk block, denoted as block_capacity, was assigned to be 10. Because of the limitation of block_capacity, a spacefilling curve of different order $p(p>1)$ was required for the different datasets. Next, for each dataset in Figures 20-(a) to (f), 5000 query points were randomly generated to make the nearest neighbor queries. Finally, we took the average of the CPU-time and the I/O time required to answer the nearest neighbor queries for each of the dataset as shown in Figures 20-(a) to (f).

\subsection{Simulation Results}

Table 2 shows that different orders for the space-filling curve, which were required for the different datasets to satisfy the limitation of block_capacity $(=10)$, since they have different data distribution. For example, for the uniform data distribution in Figure 20-(a), the space-filling curve of order 5 was required to order 10000 data points. This is because there are $1024\left(=2^{5} \times 2^{5}\right)$ disk blocks which are used to store these points and each disk block contains 10 points on the average. For the centralization, diagonal, X-parallel, and sine data distributions in Figures 20-(b), (c), (d), and (e), respectively, most of their data points are distributed in the certain region or near the certain line. Because of the limitation of block_capacity, the space-filling curve of order (5 or 6 or 7 ) was required for each of these datasets. For the real map shown in Figure 20, the space filling curve of order 6 was required to organize these data points.

First, we discuss the results of the comparison of the CPU-time of our strategies, as shown in Table 3. For all datasets, the CPU-time of the strategy based on the Peano curve is always less than that of the other two strategies. The main reason for this is as follows. For the strategy based on the Peano curve, it takes the CPU-time in function Peano_NN as shown in Figure 7. In contrast, for the strategy based on the RBG curve, in addition to the CPU-time taken in function Peano_NN, it also takes the CPU-time in the transformation between the Peano curve and itself (Figure 10). For the strategy based on 


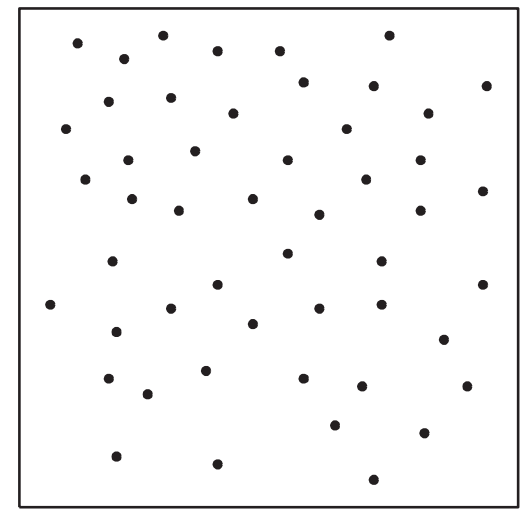

(a)

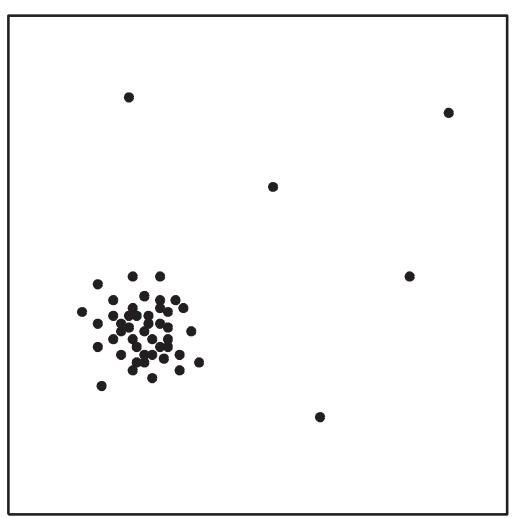

(b)

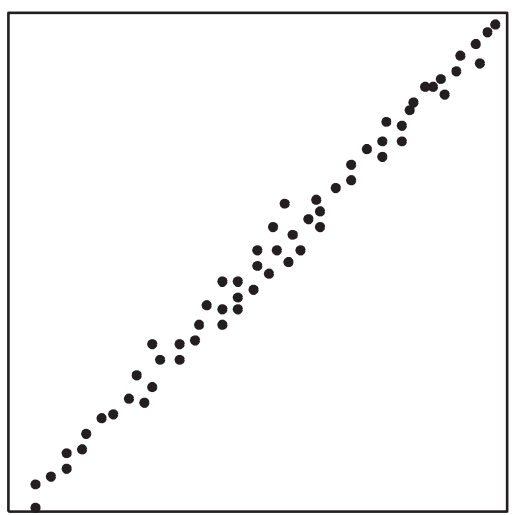

(c)

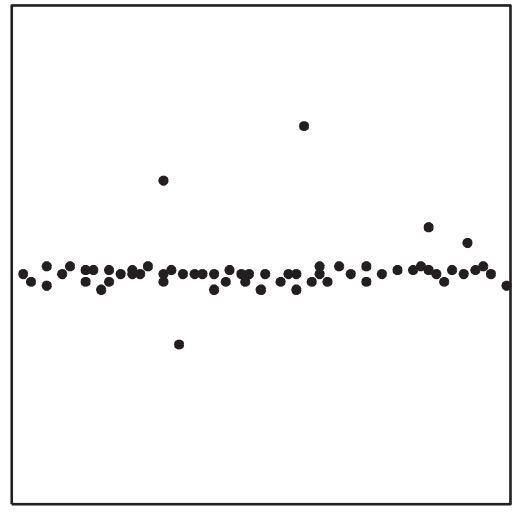

(d)

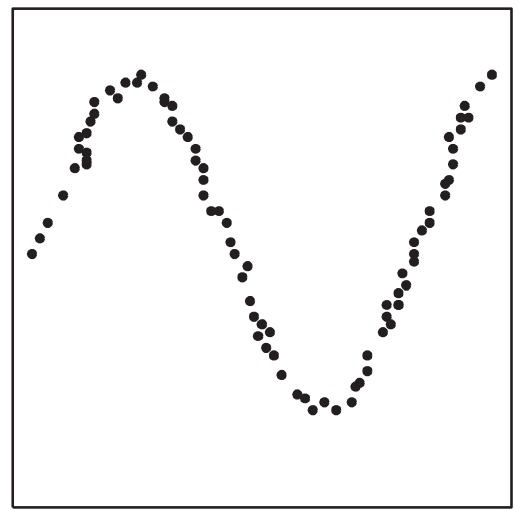

(e)

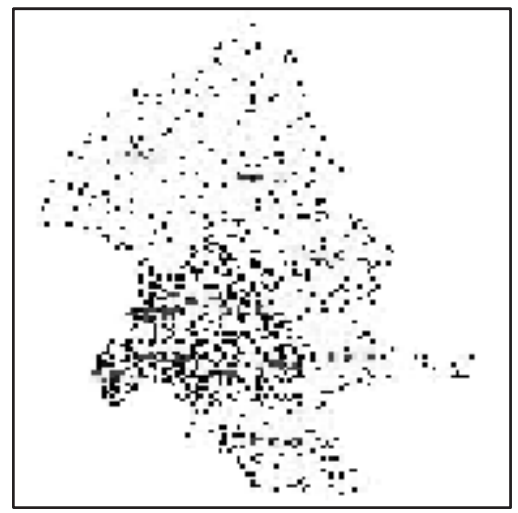

(f)

Figure 20: Six cases of data distribution: (a) uniform; (b) centralized; (c) diagonal; (d) X-parallel; (e) sine; (f) the real map. 
Table 2: Different orders for the space-filling curve to organize different datasets

\begin{tabular}{|c|c|c|c|c|c|c|}
\hline Data distribution & Uniform & Centralization & Diagonal & X-Parallel & Sine & Real Map \\
\hline order & 5 & 6 & 7 & 5 & 7 & 6 \\
\hline
\end{tabular}

the Hilbert curve, in addition to the CPU-time taken in function Peano_NN, it also takes the CPU-time in the transformation between the Peano curve and itself (Figure 12). Thus, the strategy based on the Peano curve will take less CPU-time than the strategies based on the other two curves. Therefore, for the performance measure of the CPU-time, the strategy based on the Peano curve provides better result than the strategies based on the other two curves.

Next, we discuss the effect of different datasets to the needed CPU-time. For the strategy based on the Peano curve, the CPU-time is the same among the first five different datasets. This is because the strategy based on the Peano curve uses the bit shuffling directly on the coordinates and the $z$-value to find the neighboring blocks, even though the order is greater than 1. For the real map shown in Figure 20-(f), since the data points are uniform distributed, it takes the CPU-time for the uniform distribution, which is organized by the space filling curve of order 6 .

However, for the strategy based on the RBG curve (or the Hilbert curve), the higher the order of the space-filling curve is, the longer the needed CPU-time is. It takes more CPU-time and becomes more complex than the strategy based on the Peano curve in computing the sequence numbers of the neighboring blocks next to the query block, especially when the order is increased for the different datasets. For example, since the sine data distribution requires the curve of order 7 , which is larger than the curve of order 6 required by the centralized distribution, the sine data distribution takes more CPU-time than the centralized distribution does.

Next, we discuss the performance of the I/O time among our strategies. The average positioning time for each of our three strategies on the different datasets is shown in Table 4. The transfer time for our strategies is the same, i.e., $8 * T T$. So, the average I/O time for the strategies based on the Peano curve, the RBG curve, and the Hilbert curve are $(5.3 * P T+8 * T T),(5 * P T+8 * T T)$, and $(4 * P T+8 * T T)$, respectively. In our simulation en- 
Table 3: Comparison of the CPU-time (microseconds) on different datasets

\begin{tabular}{|c|c|c|c|c|c|c|}
\hline Data distribution & Uniform & Centralized & Diagonal & X-Parallel & Sine & Real Map \\
\hline Peano & 42.0 & 48.0 & 54.0 & 42.2 & 54.0 & 50.2 \\
\hline RBG & 70.0 & 84.2 & 94.2 & 72.0 & 96.0 & 84.2 \\
\hline Hilbert & 98.2 & 114.0 & 128.2 & 100.2 & 130.2 & 112.2 \\
\hline
\end{tabular}

Table 4: Comparison of the average positioning time of I/O time for our strategies

\begin{tabular}{|c|c|c|c|c|c|c|}
\hline Data distribution & Uniform & Centralization & Diagonal & X-Parallel & Sine & Real Map \\
\hline Peano & $5 * P T$ & $5 * P T$ & $6 * P T$ & $5 * P T$ & $6 * P T$ & $5 * P T$ \\
\hline RBG & $5 * P T$ & $5 * P T$ & $5 * P T$ & $5 * P T$ & $5 * P T$ & $5 * P T$ \\
\hline Hilbert & $4 * P T$ & $4 * P T$ & $4 * P T$ & $4 * P T$ & $4 * P T$ & $4 * P T$ \\
\hline
\end{tabular}

vironment, one unit of the positioning time is average $8.8 \mathrm{~ms}$ (milliseconds), which is much longer than the CPU-time. (Note that this information is retrived from http://www.digitlife.com/articles/digests/hddreview-0602-ibm.html.) For the performance of the I/O time, our strategy based on the Hilbert curve requires less time than the strategies based on the other two curves, because the Hilbert curve can provide the better clustering property than the other two curves $[4,6,12]$. But for the performance of the CPU time, our strategy based on the Hilbert curve requires more time than the strategies based on the other two curves because of the complicated transformation rules between the Peano curve and the Hilbert curve. Since the I/O time is generally several orders of magnitude longer than the CPU time, our strategy based on the Hilbert curve can provide better performance (the CPU-time + the I/O time) than the strategies based on the Peano curve and the RBG curve for processing nearest neighbor queries.

Consequently, our strategy based on the Peano curve requires less CPU-time than the strategy based on the RBG curve (or the Hilbert curve) by using the bit shuffling property of the Peano curve. When the data is linearly ordered by the RBG curve (or the Hilbert curve), it takes most of the CPU-time in using the complicated transformation rules between the Peano curve and the RBG curve (or the Hilbert curve) to compute the sequence numbers of the 8 neighboring blocks next to the query block in the $2 D$-space. Moreover, the strategy based on the Hilbert curve requires the least total time (the CPU-time + the I/O time) in 
the nearest neighbor-finding, since the Hilbert curve provides the best clustering property among three space-filling curves.

\section{Conclusion}

In this paper, we have shown that the property of the Peano curve, bit shuffling directly on the coordinates in base 2 , is useful in directly computing the sequence number of the 8 neighboring blocks next to the query block in the $2 D$-space. Then, we have presented the transformation rules between the Peano curve and the RBG curve (or between the Peano curve and the Hilbert curve), which can be used when the data is linearly ordered by the RBG curve (or the Hilbert curve). Finally, we have compared the CPU-time and the I/O time among different nearest neighbor strategies. From our simulation, among our three strategies, we have shown that the strategy based on the Peano curve requires the least CPU-time in computing the sequence number of the 8 neighboring blocks next to the query block, and the strategy based on the Hilbert curve requires the least I/O time to access 8 neighboring blocks next to the query block because of its good clustering property. Since the I/O time is much longer than the CPU-time, the strategy based on the Hilbert curve requires the least total time (the $\mathrm{CPU}$-time + the $\mathrm{I} / \mathrm{O}$ time) to process the nearest neighbor query among our three strategies.

\section{References}

[1] A. Corral, Y. Manolopoulos, Y. Theodoridis, and M. Vassilakopoulos, "Closest Pair Queries in Spatial Databases," Proc. of ACM SIGMOD Int. Conf. on Management of Data, pp. 189-200, 2000.

[2] C. Faloutsos, "Multiattribute Hashing Using Gray Codes," Proc. of ACM SIGMOD Int. Conf. on Management of Data, pp. 227-238, Aug. 1986.

[3] C. Faloutsos, "Gray Codes for Partial Match and Range Queries," IEEE Trans. on Software Eng., Vol. 14, No. 10, pp. 1381-1393, Aug. 1988. 
[4] C. Faloutsos and S. Roseman, "Fractals for Secondary Key Retrieval," Proc. of ACM SIGACT-SIGMOD-SIGART Symp. on PODS, pp. 247-252, 1989.

[5] R. H. Guting, "An Introduction to Spatial Database Systems," Specail Issue on Spatial Database Systems of VLDB Journal, Vol. 3, No. 4, pp. 1-32, Oct. 1994.

[6] H. V. Jagadish, "Linear Clustering of Objects with Multiple Attributes," Proc. of ACM SIGMOD Int. Conf. on Management of Data, pp. 332-342, 1990.

[7] H. V. Jagadish, "Analysis of the Hilbert curve for representing two-dimensional space," Infornation Processing Letters, Vol. 62, No. 1, pp. 17-22, Apirl 1997.

[8] J. K. Lawder and P. J. H. King, "Querying Multi-dimensional Data Indexed Using the Hilbert Space-Filling Curve," ACM SIGMOD Record, Vol. 30, No. 1, pp. 19-24, Mar. 2001.

[9] S. Liao, M. A. Lopez, and S. T. Leutenegger, "High Dimensional Similarity Search With Space Filling Curves," Proc. of Int. Conf. on Data Engineering, pp. 615-622, 2001.

[10] M. F. Mokbel, W. G. Aref, and I. Kamel, "Performance of Multi-Dimensional SpaceFilling Curves," Proc. of the 10th ACM Int. Symp. on Advances in GIS, pp. 149-154, 2002 .

[11] M. F. Mokbel, W. G. Aref, I. Kamel, "Performance of multi-dimensional space-filling curves," ACM-GIS, pp. 149-154, Jan. 2002.

[12] B. Moon, H. V. Jagadish, C. Faloutsos, and J. H. Saltz, "Analysis of the Clustering Properties of the Hilbert Space-Filling Curve," IEEE Trans. on Knowledge and Data Eng., Vol. 13, No. 1, pp. 124-141, Jan. 2001.

[13] J. A. Orenstein and T. H. Merrett, "A Class of Data Structures for Associative Searching," Proc. of Symp. on PODS, pp. 181-190, 1984.

[14] J. A. Orenstein, "Spatial Query Processing in an Object-Oriented Database System," Proc. of ACM SIGMOD Int. Conf. on Management of Data, pp. 326-336, 1986. 
[15] E. M. Remgold, J. Nievergelt, and N. Deo, "Combinatorial Algorithms: Theory and Practice," Prentice-Hall Inc, Englewood Chiffs, New Jersey, 1977.

[16] B. Seeger and H. P. Kriegel, "The Buddy-Tree: An Efficient and Robust Access Method for Spatial Data Base Systems," Proc. of the 16th Conf. on VLDB, pp. 590-601, 1990.

[17] B. Seeger, P. A. Larson, and R. McFadyen, "Reading a Set of Disk Pages," Proc. of the 19th Conf. on VLDB, pp. 592-603, 1993. 


\section{Appendix A}

The source codes of the transformation rules (Procedure HilbertToPeano and Procedure PeanoToHilbert) between the Peano curve and the Hilbert curve are shown in Figure 21 and Figure 22, respectively. 
Procedure HilbertToPeano(IH);

/* Convert $h$-value to $z$-value *j

$/^{*} I H$ is a bits array that represents the binary form of the $h$-value. That is, $h_{i}, 0 \leq i \leq 2 n-1$. $^{*} /$

$/^{*} I B$ is a bits array that represents the binary form of the $z$-value. That is, $b_{i}, 0 \leq i \leq 2 n-1$. $^{*}$ /

$1^{*} I D$ is an integer array that represents the decimal form of 2 bits from left to right in $\mathrm{IH} . *$ /

/* That is $d_{j}$, for $0 \leq j \leq n-1$, for each 2-bit $h_{i} h_{i+1}$ as $s_{j}$, obtained according to the related table. */

$/ *$ IE is a temporary array that is the same array as ID. */ begin

/* Step 1: Bit Division */

$j=0$

while $j<=(n-1)$ do

begin

$I D[j]:=2 * I H[j * 2]+I H[j * 2+1]$

$I E[j]:=I D[j]$

end;

$j:=j+1$

/* Step 2: Rotate_Reflect */

$j:=0$

while $j<(n-1)$ do

begin

if $(I D[j]=0)$ then

$/ *$ Remove the right-reflection and $90^{\circ}$-clockwise rotation */

/* on the bottom-left part of the higher order curve */

begin

$k:=j+1$

while $k<=(n-1)$ do

begin

if $(I E[k]=1)$ then $I E[k]:=3$

else if $(I E[k]=3)$ then $I E[k]:=1$;

$k:=k+1$

end;

end

else

if $(I D[j]=3)$ then

$/ *$ Remove the left-reflection and $90^{\circ}$-anti-clockwise rotation */

$/ *$ on the bottom-right part of the higher order curve */

begin

$k:=j+1$

while $k<=(n-1)$ do

begin

if $(I E[k]=0)$ then $I E[k]:=2$

else if $(I E[k]=2)$ then $I E[k]:=0$;

$k:=k+1$;

end;

end;

$j:=j+1$

end;

/* Step 3: PHTransformation */

$/ *$ Change the $h$-value in the Hilbert curve to the $z$-value in the Peano curve.*/

$j:=0$

while $j<=(n-1)$ do

begin

if $(I E[j]=3)$ then $I E[j]:=2$

else if $(I E[j]=2)$ then $I E[j]:=3$;

$I B[2 * j]:=I E[j] \operatorname{div} 2$;

$I B[2 * j+1]:=I E[j] \bmod 2$

end;

$j:=j+1$

/* Step 4: Bit Concatenation */

end;

(The result is $I B$.)

Figure 21: Procedure HilbertToPeano 
Procedure PeanoToHilbert(IB);

${ }^{*}$ Convert $z$-value to $h$-value $* /$

$/^{*} I B$ is a bits array that represents the binary form of the $z$-value. That is, $b_{i}, 0 \leq i \leq 2 n-1$. $* /$

$/^{*} I H$ is a bits array that represents the binary form of the $h$-value. That is, $h_{i}, 0 \leq i \leq 2 n-1$. $^{*} /$

$/^{*} I C$ is an integer array that represents the decimal form of 2 bits from left to right in the IB. *

$/ *$ That is, $c_{j}$, for $0 \leq j \leq n-1$, for each 2 -bit $b_{i} b_{i+1}$ as $t_{j}$, obtained according to the related table. */ begin

$$
\begin{aligned}
& \text { /* Step } 6: \text { Bit Division */ } \\
& j=0 ; \\
& \text { while } j<=(n-1) \text { do } \\
& \quad \text { begin } \\
& \qquad I D^{\prime}[j]:=2 * I B[j * 2]+I B[j * 2+1] \text {; } \\
& \quad j:=j+1 ;
\end{aligned}
$$

${ }^{*}$ Change the $z$-value in the Peano curve to the $h$-value in the Hilbert curve. */

* Step \%: PHTransformation */

$j:=0$

while $j<=(n-1)$ do

begin

if $(I C[j]=3)$ then $I C[j]:=2$

else if $(I C[j]=2)$ then $I C[j]:=3$;

end $j:=j+1$

$j:=0$;

while $j<(n-1)$ do

begin

if $(I C[j]=0)$ then

$/^{*}$ Make the right-reflection and $90^{\circ}$-clockwise rotation of the lower order curve */

${ }^{*}$ to form the bottom-left part of the higher order curve */

begin

$k:=j+1$;

while $k<=(n-1)$ do

begin

$$
\begin{aligned}
& \text { if }(I C[k]=1) \text { then } I C[k]:=3 \\
& \text { else if }(I C[k]=3) \text { then } I C[k]:=1 \text {; }
\end{aligned}
$$

end; $k:=k+1$;

end

else

if $(I C[j]=3)$ then

/* Make the left-reflection and $90^{\circ}$-anti-clockwise rotation of the lower order curve */

${ }^{*}$ to form the bottom-right part of the higher order curve */ begin

$$
k:=j+1
$$

while $k<=(n-1)$ do begin

if $(I C[k]=0)$ then $I C[k]:=2$

else if $(I C[k]=2)$ then $I C[k]:=0$

$$
k:=k+1 \text {; }
$$

end; end;

$j:=j+1$;

end;

/* Step 8: Rotate_Reflect*/

$j:=0$

while $j<=(n-1)$ do

begin

$I H[2 * j]:=I C[j] \operatorname{div} 2$

$I H[2 * j+1]:=I C[j] \bmod 2$;

end;

$j:=j+1$

/* Step 9: Bit Concatenation */

end;

(The result is $I H$.)

35

Figure 22: Procedure PeanoToHilbert 